\title{
Optimización de Recorridos para la Recolección de Residuos Infecciosos
}

\author{
Silvia Simón, José Demaldé, José Hernández y Mercedes Carnero \\ Grupo de Optimización, Facultad de Ingeniería, Universidad Nacional de Rio Cuarto, \\ Campus Universitario, (5800) Rio Cuarto-Argentina (e-mail: gop@ing.unrc.edu.ar)
}

Recibido Ago. 17, 2011; Aceptado Nov. 02, 2011; Versión final recibida Feb. 21, 2012

\section{Resumen}

En este trabajo se presenta una metodología para la determinación de un conjunto de rutas óptimas para la recolección de residuos infecciosos en la ciudad de Río Cuarto, Argentina. La re colección la realizan vehículos con restricciones de capacidad. El caso puede ser formulado como un problema de programación lineal entera mixta y está catalogado como NP-duro (nondeterministic polynomial-time hard). Se propone mejorar las soluciones obtenidas mediante un algoritmo de búsqueda exacto, a través de una heurística de búsqueda local. Ésta inspecciona el entorno de dichas soluciones mediante mecanismos diferentes asegurando la explotación intensiva de las regiones promisorias del espacio de búsqueda. Se presenta la metodología y su desempeño para resolución de diferentes problemas test extraídos de la literatura y para la determinación de un conjunto de rutas óptimas.

Palabras clave: optimización, NP-duro, búsqueda local, heurísticas, recolección de residuos

\section{Optimal routes for the recollection of infectious waste}

\begin{abstract}
This paper describes a methodology to determine the optimal routes for the recollection of infectious waste in the city of Rio Cuarto in Argentina. The case can be formulated as a mixed integer linear programming problem and is classified as NP-hard. (non-deterministic polynomialtime hard). The paper proposes to improve the quality of the solutions obtained by an exact searching algorithm by mean of a local search heuristics. This technique inspects the surroundings of such solutions by different mechanisms ensuring the intensive exploitation of the promising regions of the search space. The methodology and its performance for solving different problems taken from literature and for determining a set of optimal routes are presented.
\end{abstract}

Keywords: optimization, NP-hard, local search, heuristics, waste recollection 


\section{INTRODUCCION}

Los desechos infecciosos generados en instituciones tales como hospitales y clínicas, laboratorios, veterinarias, casas de sepelios, etc. necesitan un tratamiento diferenciado debido a la peligrosidad que presentan dada la heterogeneidad de su composición, la presencia frecuente de objetos cortopunzantes y cantidades menores de sustancias tóxicas, inflamables y radioactivas de baja intensidad. En la República Argentina la ley $N^{\circ} 24051$ regula la recolección, transporte y procesamiento o disposición final de los residuos patógenos para garantizar la salud de la población hospitalaria y evitar el deterioro medio ambiental.

La gestión de los residuos infecciosos generados en centros urbanos importantes es un problema que se está abordando cada vez con mayor intensidad y que involucra varios niveles de operación: manejo y almacenamiento de los residuos dentro de la institución generadora, recolección y transporte a cargo de empresas especializadas y tratamiento definitivo de los mismos.

La ciudad de Río Cuarto, ubicada en el centro del país, tiene una población cercana a los 160000 habitantes y cuenta con más de un centenar de centros generadores de residuos infecciosos de diferente volumen de desecho. El Municipio, a través de una empresa privada, es quién se encarga de la tarea de recolección y el traslado hasta un centro provincial para su posterior tratamiento.

La recolección se realiza diariamente, atendiendo a un número de centros que varía con el transcurso de los días de la semana de acuerdo a las diferentes frecuencias de visitas que requieren cada uno de ellos. En la actualidad la planificación del recorrido se realiza sobre una base empiríca y que a través del tiempo se ha ido ajustando para tener en cuenta las variaciones del sistema.

Considerando que un análisis sistemático del problema de la recolección de residuos infecciosos puede acarrear un ahorro desde el punto de vista económico y un positivo impacto ambiental, es que se propone, en este trabajo, analizar e implementar metodologías que permitan determinar un conjunto de posibles recorridos de manera óptima.

La literatura se refiere a esta clase de problema como un Problema de Ruteo de Vehículos Períodico, PRVP. El PRVP puede pensarse como una generalización de el problema de ruteo de vehículos (PRV) convencional que intenta determinar un conjunto óptimo de rutas diarias para un período de tiempo dado. Así, resolver un PRVP lleva implícito la resolución de dos problemas básicos: un problema de planeamiento cuyo objetivo es la determinación de un conjunto de días de visita para cada centro dentro del período de tiempo que se considera y un problema de ruteo de vehículos para cada día a los fines de determinar las mejores rutas diarias, que puede ser modelado como un problema de ruteo de vehículos con restricciones de capacidad, PRVC.

En una primera etapa, se abordará el problema de optimización de los recorridos para cada día de la semana, esto es, el diseño e implementación de una metodología para la resolución de un PRVC, con el objetivo de contar con una herramienta eficiente que permita, en un trabajo futuro, resolver el problema general de la planificación y ruteo para la gestión de residuos infecciosos en Río Cuarto.

El problema de ruteo de vehículos, PRV, abarca una amplia gama de problemas que fundamentalmente consisten en encontrar un conjunto de rutas que deben ser recorridas por una flota de vehículos con el objetivo de satisfacer los requerimientos de un grupo de clientes. Dentro de ellos se encuentra el problema de ruteo de vehículos con restricciones de capacidad, en el cual, a partir de un único depósito se debe visitar un conjunto de $n$ clientes ubicados en localizaciones conocidas y que poseen determinadas demandas. Se dispone de un conjunto de $K$ vehículos, de capacidad idéntica y conocida, $Q$. El conjunto de rutas es determinado de tal manera que se optimice algún criterio de desempeño, por ejemplo, la distancia total recorrida o el costo total de transporte, sin violar la restricción de capacidad. 
Este problema ha recibido especial atención en los últimos años, debido a su gran aplicabilidad en problemas de logística y a su considerable dificultad de resolución, ya que pertenece a la clase de problemas NP-duros, (Garey y Johnson 1979; Toth y Vigo 2002). Esta última característica implica que los métodos exactos de solución se podrán aplicar solamente en los problemas de un tamaño pequeño o medio. Una recopilación de los métodos exactos utilizados hasta 2002 puede encontrarse en Thot y Vigo (2002) y más recientemente en los trabajos de Fukasawa et al. (2004, 2006), Baldacci et al. (2004) y Lysgaard et al. (2004).

Sin embargo, dado que los problemas que se presentan en las aplicaciones reales poseen un tamaño tal que los métodos exactos se tornan impracticables, se han propuesto en la literatura diversas heurísticas para la solución del PRVC, basadas en enfoques de búsqueda local, algoritmos evolutivos, recocido simulado, colonias de hormigas, etc. Así, puede citarse, por ejemplo, la denominada búsqueda local adaptiva, (Pisinger y Ropke, 2007) aplicada tanto a PRVC como a otras variantes de PRV, donde cierto número de algoritmos simples compiten para modificar la solución corriente. En cada iteración un algoritmo es elegido para destruir la solución corriente, mientras que otro es seleccionado para repararla. La nueva solución es aceptada si satisface algún criterio preestablecido.

La clásica heurística constructiva de Clark y Wright ha sido utilizada en combinación con simulación de Monte Carlo para producir un conjunto de rutas alternativas en una instancia dada del PRVC, (Juan, et al, 2010).

Dentro de los enfoques evolutivos han sido utilizados algoritmos genéticos híbridos, (Wang y Lu, 2009), que incorporan un mecanismo de barrido para mejorar la capacidad de búsqueda del algoritmo a la vez que utiliza métodos de superficie de respuestas para optimizar las probabilidades de cruzamiento y mutación; algoritmos genéticos celulares, (Alba y Dorronsoro, 2006) en los cuales la población está estructurada en una topología particular, y además el algoritmo está dotado de mecanismos de búsqueda local.

Estrategias de enjambre de partículas, con múltiples estructuras de aprendizaje social, han sido aplicadas utilizando dos tipos diferentes de representación y sus correspondientes decodificadores, (Ai y Kachitvichyanukul, 2009).

También han sido reportados algoritmos metaheurísicos poblacionales inspirados en los mecanismos de atracción-repulsión entre partículas cargadas en campos electromagnéticos, (Yurtkuran y Emel, 2010). Si bien estos algoritmos han sido utilizados en principio para problemas de optimización continuos, han sido adaptados a la resolución de problemas discretos al combinarlos con procedimientos de decodificación de las soluciones.

En este trabajo se propone mejorar un conjunto de soluciones de alta calidad obtenidas mediante un algoritmo de búsqueda exacto, a través de una heurística de búsqueda local. Ésta inspecciona el entorno de dichas soluciones mediante mecanismos diferentes y variados, asegurando la explotación intensiva de las regiones promisorias del espacio de búsqueda.

\section{MODELADO DEL PROBLEMA}

La utilización de métodos exactos para la resolución del PRVC, tales como los utilizados en el software comercial General Algebraic Modeling System, GAMS, involucra como primer paso contar con una formulación del mismo que permita su modelado en este entorno de trabajo.

A continuación se describe una formulación básica para PRVC que emplea variables binarias doblemente subindicadas, $x_{i j}$, que asumen el valor 1 si el enlace $(i, j)$ es atravesado por un vehículo en la solución óptima y el valor 0 en caso contrario. El número de tales variables es del $\boldsymbol{O}\left(n^{2}\right)$, para un problema de ruteo que deba atender exactamente a $n$ clientes.

Sea $G=(V, A)$ un grafo completamente conectado, en el cual $V=\{0,1, \ldots ., n\}$ es el conjunto de 
vértices y $A$ el conjunto de enlaces que conectan los vértices. Los vértices $i=1, \ldots, n$ corresponden a los clientes geográficamente dispersos, mientras que el nodo 0 está asociado al depósito.

El costo asociado al recorrido de un enlace cualquiera $(i, j)$ viene dado por los elementos de una matriz $\mathbf{C}$, mientras que cada elemento $q_{\mathrm{i}}$ del vector $\mathbf{q} \in \mathfrak{R}^{n}$, representa la demanda asociada al $i$ ésimo cliente.

EI PRVC formulado como un problema de programación lineal entera mixta, que incluye variables auxiliares continuas para modelar las restricciones de conectividad y capacidad, (Kara y Bektas, 2003, Kara et al, 2004), puede entonces ser escrito como:

$$
\min \sum_{i \in V} \sum_{j \in V} c_{i j} x_{i j}
$$

Sujeto a:

$$
\begin{array}{lcc}
\sum_{i \in V} x_{i j}=1 & \sum_{j \in V} x_{i j}=1 & \sum_{j \in V} x_{0 j}=K \\
\sum_{i \in V} x_{i 0}=K & ; & i=1,2, \ldots \ldots, n \\
u_{i}+\left(Q-\bar{q}_{i}-q_{i}\right) x_{0 i}-\bar{q}_{i} x_{i 0} \leq Q-\bar{q}_{i} & i=1,2, \ldots \ldots, n \\
u_{i}+\bar{q}_{i} x_{0 i} \geq q_{i}+\bar{q}_{i} & i \neq j, j=1,2, \ldots \ldots, n \\
u_{i}-u_{j}+Q x_{i j}+\left(Q-q_{i}-q_{j}\right) x_{j i} \leq Q-q_{j} & \\
x_{i j} \in\{0,1\} & \forall i, j \in V &
\end{array}
$$

Donde $r(\mathrm{~S})$ es el número mínimo de vehículos necesarios para servir a todos los vértices incluidos en $S$.

La restricción 2 impone que sólo un arco entra y deja cada vértice del grafo $G$, mientras que las ecuaciones 3 restringen a $K$ el grado asociado al depósito.

$u_{i}$ es una variable auxiliar continua que representa la carga del vehículo después de visitar al nodo i y $\bar{q}_{i}=\min _{j, j \neq i}\left\{q_{j}\right\}$

Las restricciones 4 y 5 introducen restricciones de capacidad sobre las rutas, mientras que la ecuación 6 representa tanto restricciones de capacidad como de eliminación de subrutas, esto es recorridos que no comiencen y terminen en el depósito visitando sólo una vez cada nodo.

\section{MÉTODO APROXIMADO}

La resolución exacta del PRVC utilizando paquetes comerciales es eficiente para instancias del mismo de tamaño pequeño o medio. En el caso de problemas de gran escala, como los que se encuentran en las aplicaciones reales, dicha eficiencia decae, en el sentido de poder encontrar óptimos globales en tiempos razonables. Sin embargo es posible identificar soluciones subóptimas, es decir conjuntos de rutas factibles de bajo costo, en un tiempo de cómputo aceptable.

Para ello se aplica un procedimiento de refino de tales soluciones combinando métodos heurísticos y exactos. En primer lugar se utiliza método de ramificación y corte a través del módulo CPLEX en entorno GAMS, con el objetivo de encontrar un conjunto de soluciones, en adelante conjunto de referencia, RefSet. Cada solución perteneciente al RefSet es mejorada a través de los mecanismos que se describen a continuación.

En la primera fase se realizan movimientos dentro de cada una de las rutas de la solución inicial 
con el objetivo de encontrar una secuencia de visitas con un costo menor. El problema a resolver en esta etapa puede ser asimilado al problema de viajante de comercio (Travelling Salesman Problem, TSP), ya que no deben ser tenidas en cuenta las restricciones de capacidad. Dada una ruta, esta es cortada aleatoriamente en subrutas que contienen $n c$ clientes, donde $n c$ es un parámetro del algoritmo.

La vecindad de la solución inicial está conformada por las vecindades de las rutas que la componen. La vecindad de una ruta queda definida como la colección de rutas que se obtienen al considerar todas las posibles permutaciones de clientes en las subrutas de longitud $n c$ y donde el primer y el último cliente conservan su posición.

A modo de ejemplo, considérese un conjunto de 12 clientes, con dos camiones de capacidad $Q=9500$ y demanda dada por $\mathbf{q}=\left[\begin{array}{ll}1200 & 1700150014001700140012001900180016001700\end{array}\right.$ 1100]. El método exacto proporciona la siguiente solución inicial, $\mathrm{S}_{0}$, con un costo asociado de 199, que consta de dos rutas $R 1$ y $R 2$ y donde el nodo 1 corresponde al depósito:

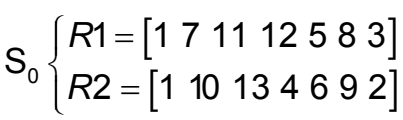

Si $n c=7$, las soluciones pertenecientes a la vecindad de $S_{0}$ tienen la siguiente estructura:

$N\left(\mathrm{~S}_{0}\right)=\left\{\begin{array}{l}N(R 1)=[1 * * * * * 3] \\ N(R 2)=[1 \bullet \cdots \bullet 2]\end{array}\right.$, donde los símbolos $* \mathrm{y} \bullet$ representan elementos pertenecientes a los conjuntos de permutaciones de los clientes (7 11 12 5 8) y (10 1346 9) respectivamente.

Las soluciones alcanzables desde $\mathrm{S}_{0}$ a través de los movimientos descriptos, se van generando y evaluando hasta que se obtiene una solución de menor costo. En el ejemplo planteado, el algoritmo abandona la fase 1 al encontrar la solución $S_{1}$, con un costo de 195:

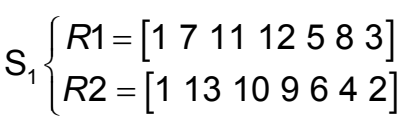

La segunda fase analiza los posibles intercambios de clientes entre rutas, para lo cual se seleccionan aleatoriamente bloques de clientes de longitud máxima lc de cada una de las rutas involucradas en la solución inicial. A continuación se procede a generar la vecindad de dicha solución definida como el conjunto de soluciones cuyas rutas contemplan todos los posibles intercambios de los bloques seleccionados entre las rutas iniciales y que además verifiquen las restricciones del problema. El valor entero lc es un parámetro del algoritmo.

Cada solución ingresa nuevamente a CPLEX ingresado como un problema modelado ahora como un TSP para inspeccionar posibles mejoras en las nuevas secuencias de visitas propuestas para cada ruta. La secuencia de transformaciones de la solución inicial, generada por CPLEX, $S_{1}$ se esquematiza a continuación. La solución $S_{2}$ es generada por la heurística descripta mientras que la solución final alcanzada $S_{3}$, con un costo de 193, es el resultado de resolver dos TSP sobre cada una de las rutas.

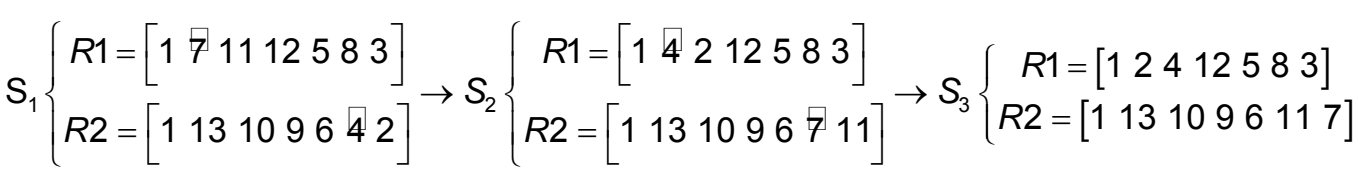

\section{EXPERIMENTOS Y RESULTADOS}

Para evaluar el desempeño de la metodología propuesta se consideró, en primer lugar, un conjunto de problemas de prueba que corresponden a diferentes instancias del PRVC. Los mismos se encuentran disponibles en Branch Cut and Price Resource Web (http://www.branchandcut.org/). Las características de cada una de las instancias resueltas se 
muestran en la Tabla 1.

Se muestran además los resultados obtenidos al resolver el problema la determinación de un conjunto de rutas óptimas en el caso de recolección de residuos infecciosos en la ciudad de Río Cuarto. En dicha ciudad están registradas como instituciones generadoras de residuos infecciosos aproximadamente 185 establecimientos. Estos presentan muy diferentes volúmenes de residuos producidos y por lo tanto las frecuencias de visitas requeridas por cada uno de ellos son variables.

En la Figura 1 se muestra la distribución geográfica de los todos los centros relevados. En este trabajo se consideró un subgrupo de 58 instituciones, las más importantes desde el punto de vista de cantidad de residuos que generan, que deben ser atendidas diariamente y para las cuales se contó con los datos de localización geográfica, y demandas diarias promedio.

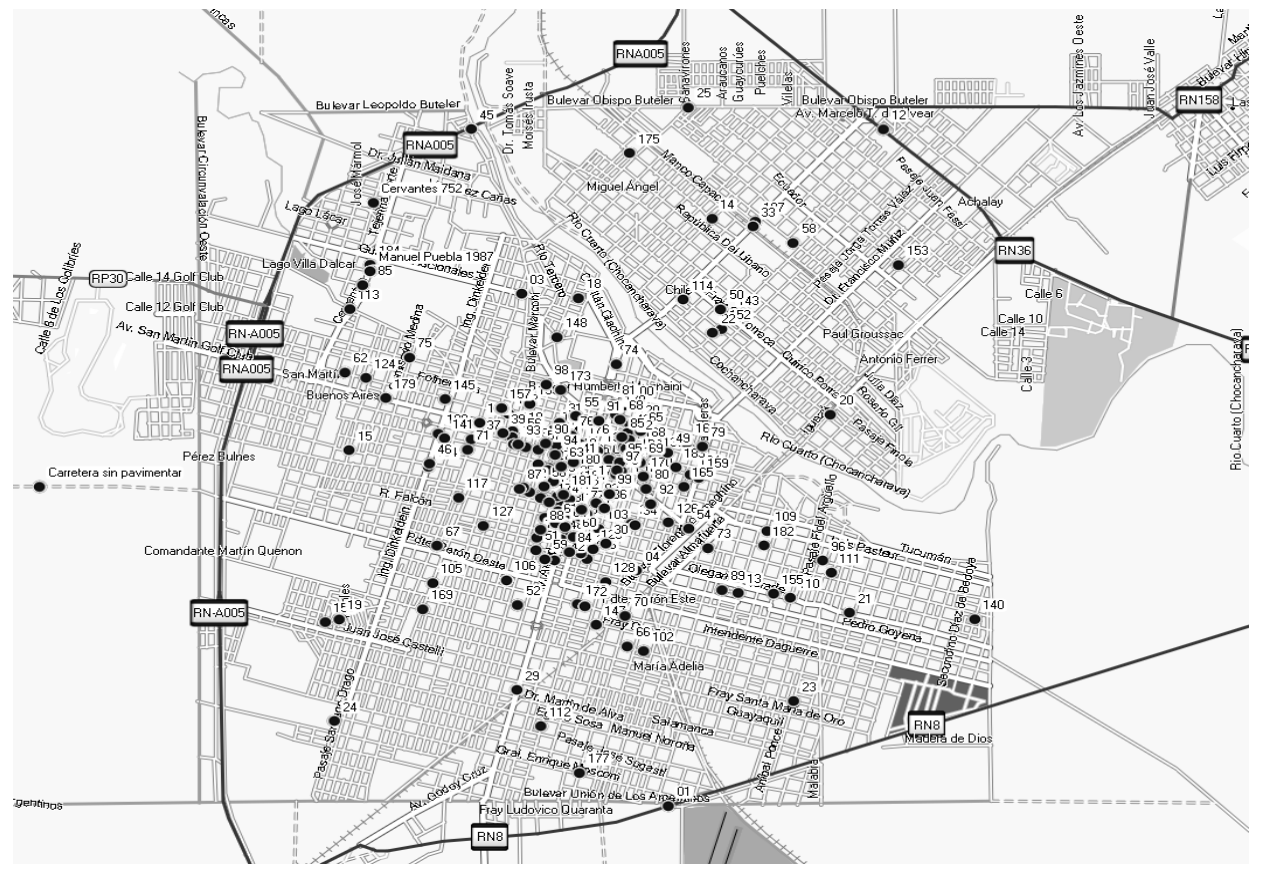

Fig. 1: Distribución geográfica de las instituciones generadoras

Se realizó un preprocesamiento de los datos de localización, para calcular las distancias mínimas de cada uno de los centros entre sí, utilizando un algoritmo de camino mínimo entre nodos. De este modo se pudo obtener una matriz de distancias, asimétrica, cuyos elementos conforman el vector de costos de la función objetivo, basando, por lo tanto, el criterio de optimización en la distancia total recorrida por cada vehículo en la ruta propuesta. Esto implica disminuir tanto el costo de combustible asociado como el desgaste de los camiones utilizados. El problema de recolección de residuos infecciosos aparece en la última fila de dicha tabla como rio59.

Tabla 1: Datos de los problemas resueltos

\begin{tabular}{|c|c|c|c|c|}
\hline Problema & $n$ & $K$ & Demanda & $\begin{array}{c}\text { Optimos de } \\
\text { referencia }\end{array}$ \\
\hline \hline eil13 & 12 & 4 & Variable & 247 \\
\hline gr17 & 16 & 3 & Uniforme & 2685 \\
\hline p-n21-k2 & 20 & 2 & Uniforme & 211 \\
\hline bays29 & 28 & 5 & Uniforme & 2963 \\
\hline eil31 & 30 & 7 & Variable & 379 \\
\hline p-n76-k4 & 75 & 4 & Variable & 593 \\
\hline p-n101-k4 & 100 & 4 & Variable & 681 \\
\hline rio59 & 58 & 2 & Variable & --- \\
\hline
\end{tabular}

Las soluciones óptimas han sido alcanzadas en todos los problemas test, cuando se utilizó el método aproximado como estrategia de resolución, con parámetros $n c \in[57]$ y $l c=5$.

El equipamiento utilizado para realizar los calculos es un computador Intel Q6600 de cuatro 
núcleos 2.4 GHz, 2GB de RAM, bajo sistema Operativo Windows XP con SP3. Los prototipos de los algoritmos implementados han sido desarrollados en Matlab 7, para el sistema operativo mencionado.

En el caso del problema de aplicación, rio59, el mejor valor encontrado de función objetivo, con el método aproximado, fue de 71.9 , en un tiempo total de 61.7 segundos correspondiente a las siguientes rutas:

Ruta \# 1: 53-27-33-29-35-7-39-25-8-55-26-9-40-46-43-34-4-50-51-28

Ruta \# 2: 1-23-18-14-45-38-17-2-16-44-24-11-13-32-57-49-21-19-15-48-36-10-41-54-30-42$56-37-31-52-5-6-47-58-3-12-20-22$

\section{CONCLUSIONES}

En este trabajo se diseñó e implementó un método aproximado para la resolución del problema de ruteo de vehículos con restricción de capacidad. Dicho método fue probado en un conjunto de problemas test con la finalidad de establecer la calidad de las soluciones obtenidas por la heurística, sus posibilidades de escalamiento y el esfuerzo computacional asociado.

Para el conjunto de problemas seleccionados, el comportamiento de la heurística ha sido satisfactorio si se comparan los tiempos de cómputo insumidos para refinar la solución inicial así como la calidad de la solución final obtenida.

En el problema de aplicación seleccionado se pudo arribar a una muy buena solución en un tiempo sensiblemente menor que el requerido por un método exacto. Esto hace que las posibilidades de abordar con éxito problemas de mayor envergadura con el método aproximado aparezcan como muy promisorias.

\section{REFERENCIAS}

$\mathrm{Ai}$, J., V. Kachitvichyanukul; Particle swarm optimization and two solution representations for solving the capacitated vehicle routing problema, Computers \& Industrial Engineering 56, 380387 (2009).

Alba, E., B. Dorronsoro; Computing nine new best-so-far solutions for Capacitated VRP with a cellular Genetic Algorithm. Information Processing Letters 98 225-230 (2006).

Alkın Yurtkuran, A.; y E. Emel A new Hybrid Electromagnetism-like Algorithm for capacitated vehicle routing problems Expert Systems with Applications ,37, 3427-3433 (2010).

Baldacci, R., E. Hadjiconstantinou y A. Mingozzi; An exact algorithm for the capacitated Vehicle routing problem based on a two-commodity network flow formulation, Operations Research 52, pp. 723-738 (2004).

Fukasawa, R., H. Longo, J. Lysgaard, D. Poggi, M. Reis, E. Uchoa y R. Werneck; Robust branchand-cut-and-price for the capacitated vehicle routing problem. Math. Program. Ser. 106, 491-511 (2006).

Fukasawa, R., J. Lysgaard, D. Poggi, M. Reis, E. Uchoa y R. Werneck Robust branch-and-cutand-price for the capacitated vehicle routing problem. Proceedings of the X IPCO, Lecture Notes in Computer Science 3064, 1-15 (2004).

Garey, M. y Johnson, D., "Computers and Intractability. A guide to the theory of NPCompleteness, W.H. Freeman and Company, New York (1999).

Juan, A.; Faulin, J.; Ruiz, R.; Barrios, B. Y S. Caballe; The SR-GCWS hybrid algorithm for solving the capacitated vehicle routing problem. Applied Soft Computing 10, 215-224 (2010). 
Kara, I.,T. Bektas; Integer Linear Programming Formulation of the Generalized Vehicle Routing Problem, EURO/INFORMS Joint International Meeting, July 06-10, Istanbul, Turkiye (2003).

Lysgaard, J., A. Letchford y R. Eglese; A new branch-and-cut algorithm for the capacitated vehicle routing problem. Mathematical Programming, Series A;100, 423-45 (2004).

Pisinger, D; S. Ropke; A general heuristic for vehicle routing problems. Computers \& Operations Research 34, 2403 - 2435 (2007).

Toth, P., D. Vigo; An overview of vehicle routing problems. In P. Toth \& D. Vigo (Eds.). Philadelphia: Siam (2002).

Wang, C.H., y J. Lu; A hybrid genetic algorithm that optimizescapacitated vehicle routing problem. Expert Systems with Applications, 36,2921-2936 (2009). 